\title{
Estudio comparativo de costos en el tratamiento de cáncer de próstata Centro Médico Naval, 2008-2011
}

\section{Comparative study of costs in the treatment of prostate cancer Naval Medical Center, 2008-2011}

\author{
Oscar Quevedo ${ }^{1}$, Víctor Alarcón ${ }^{2}$, Jesús Rumiche ${ }^{3}$, Miguel Benito ${ }^{4}$
}

Recibido: 07/11/2019 Aceptado: 11/12/2020 Publicado: 31/12/2020

\begin{abstract}
Resumen
El tratamiento farmacológico con medicamentos biológicos para enfermedades oncológicas significa una alta inversión para el Perú; por tal motivo, es de suma importancia utilizar racionalmente los recursos realizando diversos estudios fármaco-económicos. El objetivo fue determinar y comparar la relación costo- efectividad de acetato de leuprolide 7,5 mg frente a goserelina 3,6 mg en el Centro Médico Naval "Cirujano Mayor Santiago Távara", Lima, Perú. Es un estudio farmacoepidemiológico de corte farmacoeconómico, con diseño analítico y retrospectivo. Se revisaron y analizaron las historias clínicas de 46 pacientes con cáncer de próstata durante el período de enero 2008 a diciembre 2011, a quienes se les prescribió uno de los fármacos en estudio. Se determinó la efectividad, el costo medio de la terapia oncológica por paciente y el costo-efectividad. La información se procesó utilizando el programa estadístico SPSS versión 22, obteniendo: frecuencias, medias, desviación estándar, error estándar y coeficiente de variación e inferencial (t student) con intervalo de confianza $95 \%, p<0,05$. Se halló alta efectividad expresada como reducción de antígeno prostático específico (PSA) en leuprolide $(85,84 \%)$ y goserelina $(90,72 \%)$, diferencia que no fue estadísticamente significativa $(p>0,05)$. El fármaco que obtuvo la menor relación costo-efectividad por porcentaje de reducción de PSA fue goserelina $(S / .73,69)$ frente a acetato de leuprolide (S/.155,27). Se puede concluir que la mejor alternativa costo-efectiva estimada en el tratamiento del cáncer de próstata en el Centro Médico Naval durante el periodo de enero 2008 a diciembre 2011 fue goserelina.
\end{abstract}

Palabras clave: Estudio fármaco-económico; Acetato de leuprolide; goserelina; cáncer de próstata; antígeno prostático específico; costo-efectividad.

\begin{abstract}
The pharmacological treatment with biological drugs for oncological diseases means a high investment for Peru, for this reason, it is of the utmost importance to rationally use the resources by conducting the various pharmaco-economic studies that exist. The objective was to determine and compare the cost-effectiveness ratio of leuprolide acetate $7.5 \mathrm{mg}$ and goserelin $3.6 \mathrm{mg}$ in Naval Medical Center "Surgeon Major Santiago Távara", Lima, Peru. It is a pharmacoepidemiological study of a pharmaco-economic nature. Analytical, retrospective design. The medical records of 46 patients diagnosed with prostate cancer during the period from January 2008 to December 2011, who were prescribed one of the study drugs were reviewed and analyzed. The effectiveness, average cost of cancer therapy per patient and cost-effectiveness were determined. The information was processed using the statistical program SPSS version 22, obtaining frequencies, means, standard deviation, standard error and coefficient of variation and inferential (t student) with $95 \%$ confidence interval, $\mathrm{p}<0.05$. It was found high effectiveness expressed as a percentage reduction in prostate specific antigen, $85.84 \%$ with leuprolide acetate and $90.72 \%$ with goserelin, a difference that was not statistically significant

1 Universidad Nacional Mayor de San Marcos, Facultad de Farmacia y Bioquímica. Lima, Perú. E-mail: quevedo.oscar1@gmail.com

2 Universidad Nacional Mayor de San Marcos, Facultad de Farmacia y Bioquímica. Lima, Perú. E-mail: vaag-08@hotmail.com

3 Universidad Nacional Mayor de San Marcos, Facultad de Farmacia y Bioquímica. Lima, Perú. Autor para correspondencia: jrumicheb@unmsm.edu.pe

4 Universidad Nacional Mayor de San Marcos, Facultad de Medicina, Departamento Académico de Medicina Preventiva y Salud Pública. Lima, Perú.

E-mail: mbenitom@unmsm.edu.pe
\end{abstract}

\section{Citar como:}

Quevedo, O., Alarcón, V., Rumiche, J. y Benito, M. (2020). Estudio comparativo de costos en el tratamiento de cáncer de próstata Centro Médico Naval, 2008-2011. Ciencia e Investigación 2020 23(2):3-8. doi: http://dx.doi.org/10.15381/ci.v23i2.19375

(C) Los autores. Este artículo es publicado por la Ciencia e Investigación de la Facultad de Farmacia y Bioquímica de la Universidad Nacional Mayor de San Marcos. Este es un artículo de acceso abierto, distribuido bajo los términos de la licencia Creative Commons Atribucion - No Comercia_Compartir Igual 4.0 Internacional. (http://creativecommons.org/licenses/by-nc-sa/4.0/) que permite el uso no comercial, distribución y reproducción en cualquier medio, siempre que la obra original sea debidamente citada. 
$(p>0.05)$. The drug that obtained the lowest cost-effectiveness ratio by percentage reduction of prostate specific antigen was gosereline (S/. 73.69) compared with leuprolide acetate (S/. 155.27). It was concluded that the most estimate cost-effective alternative in the treatment of prostate cancer at the Naval Medical Center during the period from January 2008 to December 2011 was goserelin.

Keywords: Pharmacoeconomic study; leuprolide acetate; goserelin; prostate cancer; prostate-specific antigen; cost-effectiveness.

\section{INTRODUCCIÓN}

El cáncer de próstata es un problema de salud pública en el mundo, que se presenta principalmente en hombres de edad avanzada; casi dos tercios de los casos de cáncer de próstata se diagnostican en hombres de 65 años o más; siendo 67 años la edad promedio al momento de realizarse el diagnóstico. Es el cáncer más común, después del cáncer de piel ${ }^{1-3}$.

En 2015 se diagnosticó cáncer prostático a 220,800 varones en Estados Unidos y 27,540 murieron a causa de este tumor en ese mismo año ${ }^{4}$.

Los datos en el Centro Médico Naval (CMN) revelan que la incidencia de cáncer de próstata se ha incrementado en un $48 \%$ entre los ańos 2008 y 2010, representando $32 \%$ del total de cánceres registrados en el año 2010 en hombres 5 .

Se han descrito diversos tipos de tratamiento para esta enfermedad, dependiendo del riesgo de recidiva y la esperanza de vida. En una primera opción y cuando el cáncer de próstata es localizado y el riesgo de recidiva es bajo, la indicación es la prostatectomía radical ${ }^{6}$. Igual comportamiento se espera si el riesgo es bajo e intermedio y la esperanza de vida es superior a los 10 años. La supervivencia específica a 10 años es superior al $94 \%{ }^{7}$. En Cáncer de Próstata de alto riesgo, la Prostatectomía Radical es opcional, especialmente en pacientes jóvenes y en el contexto de un tratamiento multimodal. Debido a la elevada probabilidad de márgenes quirúrgicos (33$66 \%)$ y ganglios positivos (8-49\%), entre el 56 y el $78 \%$ de los pacientes precisan tratamiento adyuvante o de rescate con radioterapia o supresión androgénica. La supervivencia específica a 5, 10 y 15 años es del 95, 90 y $79 \%$, respectivamente ${ }^{7}$.

La segunda opción es la disminución de la testosterona plasmática que puede lograrse inhibiendo la producción a través del eje hipotálamo-hipófisis-testículo o mediante el bloqueo periférico con inhibidores competitivos de la unión andrógeno-receptor ${ }^{7}$. El tratamiento inicial recomendado en la práctica clínica se realiza a través de la inhibición de la producción hormonal mediante castración quirúrgica (Orquiectomía subalbugénea) o lo que es mucho más frecuente mediante el empleo de agonistas de la hormona liberadora de la hormona luteinizante $(\text { aLHRH })^{8-9}$.

La orquiectomía consigue un rápido descenso de la testosterona plasmática por debajo de $20 \mathrm{ng} / \mathrm{mL}$. Tiene ventajas por su bajo costo económico, rapidez en conseguir el objetivo y evita la falta de adherencia al tratamiento; pero también tiene desventajas: el impacto emocional, complicaciones derivadas del acto quirúrgico y la irreversibilidad del procedimiento 9 .

Las ventajas de los análogos LHRH incluyen su eficacia, su excelente perfil de tolerancia, su comodidad (un vial trimestral), el menor impacto psicológico que producen al paciente frente a la cirugía de castración y la posibilidad de reversibilidad del bloqueo. Al frente de sus desventajas están su elevado precio y la estimulación de testosterona durante las 3-4 primeras semanas (fenómeno flare), lo que puede provocar una exacerbación de los síntomas o la aparición de complicaciones como la compresión medular en caso de metástasis óseas lumbares. Para evitar este fenómeno es obligada la adición de un anti andrógeno periférico durante las primeras semanas con el fin de bloquear el exceso de testosterona circulante?.

La goserelina y la leuprorelina son dos de las cuatro formulaciones de análogos existentes en el mercado (triptorelina y buserelina) con preparaciones de liberación retardada que requiere la administración subcutánea o intramuscular en un rango de frecuencia de 1 a 3 meses. Un metaanálisis que recoge más de 10 ensayos clínicos controlados que han incluido a cerca de 2000 pacientes ha demostrado que la eficacia de estas moléculas es comparable con la castración quirúrgica ${ }^{7}$.

El tratamiento farmacológico con medicamentos biológicos para enfermedades oncológicas significa una alta inversión para el sistema de salud de los países, y el Perú no es la excepción ${ }^{\mathbf{1 2}}$; tal como lo demuestra un estudio de costos de medicamentos oncológicos realizado por el Ministerio de Salud en el año 2011, donde el costo del fármaco goserelina 3,6 mg era de S/ 7980 y para leuprorelina 2,8 $\mathrm{mg}$ (leuprolina 2,8 mg) de S/640, por cada ampolla ${ }^{8}$.

En nuestro país, donde se asigna un presupuesto limitado para los gastos de salud, es importante racionalizar los recursos para lo cual, este tipo de estudio fármaco económico es importante 9 , considerando que esta enfermedad es una de las más prevalentes en varones debido a que con el pasar de los años el número de casos se está incrementando y que el tratamiento es de costo elevado, por lo que nos planteamos como objetivo determinar y comparar la relación costo - efectividad de leuprolide 7,5 mg y goserelina 3,6 mg en el tratamiento de cáncer de próstata. 


\section{MATERIALES Y MÉTODOS}

Tipo de estudio. Es un estudio de corte fármaco-económico, que sigue un diseño observacional, retrospectivo y comparativo

\section{Población y muestra}

Población: 135 historias clínicas (HC) de pacientes hospitalizados y ambulatorios, con edades entre 50 a 79 años y nivel de PSA mayor a $4 \mathrm{ng} / \mathrm{mL}$ que asistieron a los consultorios externos de los servicios de Urología u Oncología, diagnosticados con cáncer de próstata entre enero 2008 y diciembre 2011, en el Centro Médico Naval "Cirujano Mayor Santiago Távara” (CMN “CMST”) y a quienes se les prescribió acetato de leuprolide $7,5 \mathrm{mg}$ o goserelina 3,6 mg.

Muestra: Se obtuvieron $23 \mathrm{HC}$ de pacientes con indicación de acetato de leuprolide $7,5 \mathrm{mg}^{9}$ y 23 historias clínicas de pacientes con indicación de goserelina 3,6 mg ${ }^{\mathbf{1 0}}$.

Se aplicaron los siguientes criterios de inclusión y exclusión,

a. Criterios de inclusión: 1. HC de pacientes diagnosticados con cáncer de próstata con inicio o reinicio de tratamiento con los fármacos en estudio, en el período entre enero 2008 y diciembre 2011 cuyas edades fluctúen entre 50 y 79 años; 2 . HC de pacientes con inicio de terapia farmacológica de bloqueo androgénico total junto con bicalutamida $150 \mathrm{mg}$ y que mínimamente hayan recibido tratamiento continuo ( 2 a 6 meses) con leuprolide 7,5 mg o goserelina $3,6 \mathrm{mg}$.

b. Criterios de exclusión: 1. HC de pacientes sometidos a procedimiento quirúrgico (orquiectomía, prostectomía, radioterapia u otros similares) previo o paralelamente al tratamiento con los fármacos de estudio; 2. HC de pacientes que interrumpieron el tratamiento antes de cumplir 2 meses de administración continúa con los fármacos en mención; 3. $\mathrm{HC}$ de pacientes que no iniciaron la terapia farmacológica de bloqueo androgénico total junto con bicalutamida $150 \mathrm{mg}$ o que hayan iniciado o estén recibiendo flutamida $250 \mathrm{mg}$ y 4 . HC que no presenten resultados de PSA basales y no reporten control serológico de PSA en ningún período después del inicio o reinicio del tratamiento.

\section{Metodología}

1. Recopilación de datos a partir de las historias clínicas: Se seleccionaron 135 historias clínicas del servicio de Bioestadística del CMN "Cirujano Mayor Santiago Távara”, aplicando criterios de inclusión de pacientes diagnosticados con cáncer de próstata.

Aplicando criterios de exclusión se obtuvieron 46 historias clínicas, que se distribuyeron en 2 grupos:

- Grupo "A": con prescripción de acetato de leuprolide $7,5 \mathrm{mg}(\mathrm{n}=23)$.

- Grupo “B”: con prescripción de goserelina 3,6 $\operatorname{mg}(n=23)$.
Los datos relevantes de esta investigación se trasladaron a la ficha de recolección de datos

\section{Consumo y costo de los medicamentos en la tera- pia oncológica}

El consumo de medicamentos de cada paciente que se seleccionó, se analizó utilizando los reportes de consumo mensual y anual de la Farmacia Onconaval del CMN "CMST", calculando el costo que representó para el hospital el tratamiento farmacológico.

\section{Análisis costo-efectividad ${ }^{11}$.}

a. Determinación de la efectividad. Expresada en porcentaje promedio de reducción de niveles séricos de PSA; valores menores a $4,0 \mathrm{\eta g} / \mathrm{mL}$, que es lo buscado para alcanzar el objetivo terapéutico, obtenido a partir de los 2 meses para los dos bloques de tratamiento.

b. Determinación del costo medio de la terapia oncológica por paciente. Se determinó inicialmente el costo total para cada medicamento que resultó de la suma de los valores que se obtuvieron de multiplicar el número de unidades inyectables por su respectivo costo. Luego, estos resultados se dividieron entre el número de pacientes correspondientes para cada grupo, obteniéndose el costo medio por paciente.

c. Determinación del costo-efectividad. Se obtuvo dividiendo b/a es decir, el costo medio por paciente entre la efectividad (el \% de reducción medio de PSA) para los grupos con ambos medicamentos,

d. Comparación entre ambos grupos. Se digitó en una base Excel y se procesó utilizando el programa estadístico SPSS versión 22. El análisis fue de tipo descriptivo (frecuencias, medias, desviación estándar, error estándar y coeficiente de variación) referido a los valores de PSA e inferencial (t de student).

\section{RESULTADOS}

En ambos grupos, no se encontraron diferencias estadísticamente significativas $(p>0,05)$ al analizar los valores iniciales de PSA $(17,653$ y $15,583 \mathrm{jg} / \mathrm{mL}$ para leucoprolide y goserelina respectivamente), sin embargo los valores de finales, de $1,321 \mathrm{ng} / \mathrm{mL}$ y $0,823 \mathrm{jg} / \mathrm{mL}$, para leuprolide y goserelina respectivamente, presentaron diferencias estadísticamente significativas $(\mathrm{p}<0,05)$.

El gasto generado por el consumo de medicamentos oncológicos en el CMN "CMST" es más elevado para el acetato de leuprolide $7,5 \mathrm{mg}$, correspondiéndole S/. 306 559.00 , comparado con la goserelina $3,6 \mathrm{mg}$ cuyo gasto fue S/. 153 772.50. (Tabla 1). Asimismo, la goserelina presenta el menor "costo medio por paciente".

En relación al costo-efectividad de acetato de leuprolide 7,5 mg y goserelina 3,6 mg, se encuentra que éste último presenta goserelina 3,6 mg presenta menor relación 
costo-efectividad, 73,69 nuevos soles por porcentaje de reducción de PSA, en comparación con acetato de leuprolide $7,5 \mathrm{mg}$ (Tabla 2 y figura 1 ).

\section{DISCUSIÓN}

La Dirección General de Epidemiología reporta en el $2015^{12}$ que los cánceres más frecuentes en el sexo masculino son de próstata $(15,1 \%)$, de estómago (11,1\%) y de piel $(7,8 \%)$. Según DIGEMID para el $2014^{13}$ las compras oncológicas del sector público eran el $98 \%$. Asimismo, en el 2019, se considera como problema sanitario el cáncer y se señala como una de las prioridades de investigación ${ }^{14}$, el estudio de la magnitud, distribución, sobrevida e impacto económico, social e individual del cáncer en el Perú.

El presente estudio es retrospectivo de análisis fármaco-económico, en el que se comparó el costo-efectividad del uso de acetato de leuprolide $7,5 \mathrm{mg}$ frente a goserelina 3,6; para ello se revisaron 135 historias clínicas; sin embargo, uno de las limitaciones encontradas fue que en muchas de ellas no se reportaban valores iniciales de PSA, o, de lo contrario dicha información no clara y ordenada, por lo que se tuvieron que descartar.

El análisis fármaco-económico que se desarrolló fue de costo- efectividad media considerando los efectos sobre la salud, por lo que se mide el porcentaje de reducción de PSA. Otros análisis fármaco-económicos miden diferentes parámetros, así el análisis de minimización de costo puede medir eficacia clínica y seguridad ${ }^{15}$, existen análisis de costo-efectividad que miden datos de supervivencia ${ }^{16}$ o calidad de vida relacionada con la salud. Los efectos sobre los recursos para todos los análisis se miden en unidades monetarias.

Se concluyó que la efectividad entre acetato de leuprolide y goserelina es similar (Tabla 1), ambos en combinación con bicalutamida, sin embargo se recomienda hacer más estudios para identificar diferencias clínicas significativas entre los diferentes agonistas, tal como lo considera Bolton et al. ${ }^{17}$ Iannazzo et al. ${ }^{16}$ en un metaanálisis mostraron que los niveles de testosterona más

Tabla 1. Costo total de fármacos oncológicos prescritos por tiempo de tratamiento

\begin{tabular}{lcc}
\hline Costos & Grupo con indicación de acetato de leuprolide $\mathbf{7 , 5} \mathbf{~ m g ~ ( n = 2 3 )}$ & Grupo con indicación de goserelina 3,6 mg (n=23) \\
& $(\mathbf{S} /)$. & 668,76 \\
\hline Costo medio por paciente & 13328,65 & 153772,50 \\
Costo del consumo total & 306559,00 & 150 \\
\hline
\end{tabular}

Tabla 2. Análisis costo-efectividad de fármacos oncológicos prescritos

\begin{tabular}{ccc}
\hline & $\begin{array}{c}\text { Grupo con indicación de acetato de } \\
\text { leuprolide }(\mathrm{n}=23)\end{array}$ & $\begin{array}{c}\text { Grupo con indicación de goserelina } \\
(\mathrm{n}=23)\end{array}$ \\
\hline Costo/paciente (nuevos soles) & 13328,65 & 685,76 \\
Efectividad (media \% reducción PSA) & 85,84 & 90,72 \\
Costo-efectividad (soles por porcentaje de reducción de PSA) & 155,27 & 73,69 \\
\hline
\end{tabular}

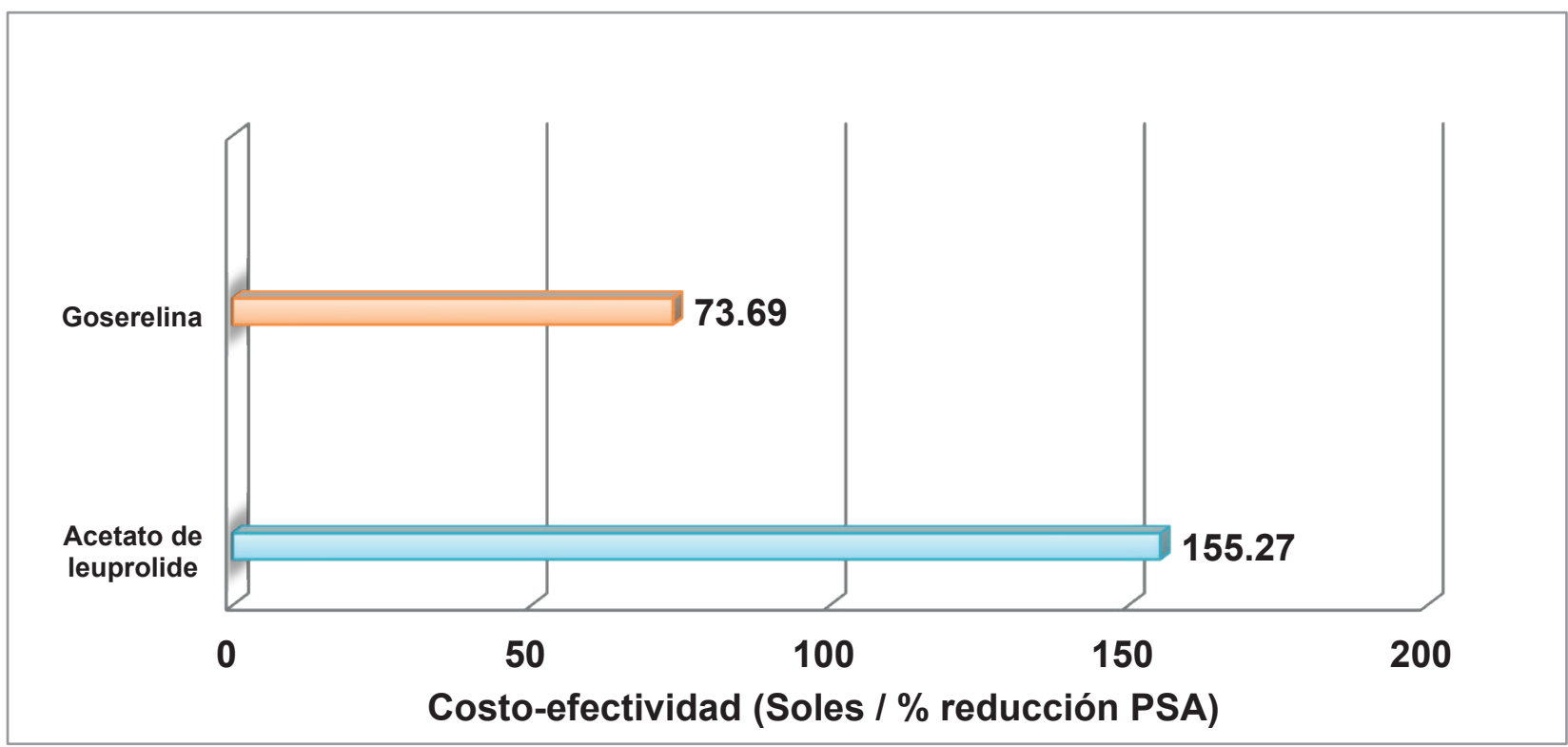

Figura 1. Costo efectividad de fármacos oncológicos prescrito 
bajos se lograron con buserelina y leuprorelina $22,5 \mathrm{mg}$ (8-9 ng/dL) comparado con lo encontrado cuando se administra con goserelina (18 ng/dL). Reis et al. ${ }^{18}$, en su estudio encuentran que 3 meses después de la primera inyección, los pacientes con cáncer de próstata metastásico tratados por un mes con goserelina tuvieron disminución significativa de PSA comparado con los pacientes tratados con leuprorelina 3,75 o $7,5 \mathrm{mg}$ por un mes; sin embargo, no hubo correlación entre la reducción de los niveles de PSA y la reducción de testosterona, por lo que sugirieron hacer estudios a mayores escalas para determinar si la goserelina es más efectiva.

La medida de testosterona sérica podría haber sido otra forma de comparar la efectividad del tratamiento; pero el CMN no realiza dicha prueba, sin embargo es importante considerar que Fujii et al. ${ }^{19}$ en un estudio retrospectivo compararon los efectos de ambos fármacos en pacientes japoneses con edad promedio de 69,8 años y nivel de testosterona previo al tratamiento con LHRH de $4,54 \mathrm{ng} / \mathrm{mL}$. Y reportaron como niveles máximos de testosterona durante los tratamientos de: leuprolide por un mes ( 40 pacientes) y por tres meses (68), goserelina por un mes (50) y por tres meses (74) los siguientes valores: 0,$22 ; 0,20 ; 0,19$ y $0,20 \mathrm{ng} / \mathrm{mL}$ respectivamente (no significativo), concluyendo que las formulaciones de uno y tres meses de leuprolide y goserelina tienen efectos equivalentes y suficientes para suprimir los niveles séricos de testosterona en el cáncer de próstata. Cheng et al. ${ }^{20}$ evaluaron la terapia de goserelina y encontraron una mejor sobrevivencia y mayores años de vida ponderados por la utilidad y fue más costo-efectiva que otras quimioterapias.

El tratamiento de esta enfermedad representa un alto costo para los pacientes; y dicho costo tiende aumentar a través del tiempo y es asumido por la institución, por lo que tiene impacto significativo en el sistema de salud del país y en la economía de los hogares. La orquiectomía resulta ser la estrategia costo-efectiva más apropiada, pero los pacientes generalmente rechazan la castración quirúrgica. En Italia, Iannazzo et al. ${ }^{16}$ para establecer la mejor opción costo-efectiva entre los agonistas LHRH disponibles en el Servicio Nacional de Salud Italiano (SNIS), realizaron un metaanálisis fármaco-económico comparando tratamientos de tres meses concluyendo que la leuprorelina $22,5 \mathrm{mg}$ es la más costo-efectiva de las formulaciones depot de los agonistas LHRH considerando la supervivencia, resultado diferente al encontrado en el presente estudio, donde la que presentó menor relación costo-efectividad fue goserelina 3,6 mg; sin embargo se debe considerar que la dosificación aplicada en dicho estudio fue la trimestral, en tanto que en el presente estudio se utilizó la dosificación mensual la cual era de mayor uso o prescripción en el CMN "CMST". No se encontraron diferencias estadísticamente significativas en la reducción y porcentaje de reducción de los niveles de PSA, entre los grupos de pacientes con prescripción de leuprolide $7,5 \mathrm{mg}$ con los que recibieron goserelina $3,6 \mathrm{mg}$.

\section{CONCLUSIONES}

Se puede concluir que goserelina 3,6 mg presenta una menor relación costo-efectividad comparada con acetato de leuprolide $7,5 \mathrm{mg}$.

\section{AGRADECIMIENTOS}

Al Q.F. Maykel Centeno Romero y a todas las personas del Centro Médico Naval "Cirujano Mayor Santiago Távara", que mediante su apoyo desinteresado permitieron la ejecución del presente proyecto. Este artículo corresponde a un trabajo de tesis de la Facultad de Farmacia y Bioquímica. UNMSM.

\section{REFERENCIAS BIBLIOGRÁFICAS}

1. National Cáncer Institute, Tipos comunes de cáncer en la población hispana [Sede Web], [Acceso 09 de agosto de 2013].

2. Disponible en: http://www.cancer.gov/espanol/tipos/comunes-hispanos.

3. Cruz G, Carballo E, Valdivia D, Massip N, García H. Mortalidad por tumores malignos más frecuentes en el adulto mayor. Rev Cubana Med Gen Integr. 2011, 27(1): 83-90.

4. Ministerio de Salud. Cifras nacionales de cáncer. Lima: MINSA; 2010.

5. Harrison's Principles of Internal Medicine Harrison. (APA) (2016). Harrison, Manual de Medicina Interna [Traducción al español de Harrison's Principles of Internal Medicine]. (19a edición). México. Página 342.

6. Centro Médico Naval. Servicio de anatomía patológica; Programa Médico Onconaval; Incidencia de cáncer Hospital Naval años 1994-2012. Lima: CMN; 2013.

7. D'Amico AV, Whittington R, Malkowicz S, Schultz D, Blank K, Broderick G, et al. Biochemical outcome after radical prostatectomy, external beam radiation therapy, or interstitial radiation therapy for clinically localized prostate cancer. JAMA. 1998; 280:969-74.

8. Heidenreich A, Bastian P, Bellmunt J, Bolla M, Joniau S, van der Kwast T, et al. EAU guidelines on prostate cancer. Part 1: Screening, diagnosis, and local treatment with curative intent-update 2013. Eur Urol. 2014;65:124-37.

9. Rodríguez-Antolín A, Leiva Galvis O. Modalidades de supresión hormonal en el cáncer de próstata avanzado. Revista Médica. 2005; 52: 78-81.

10. Asociación Española de Urología APA (APA) (2007) Libro del Residente de Urología (1ra edición). España. Página 557.

11. Cortijo D, Foneron A, Troncoso L, Ebel L, Gil G, Valdevenito G, Vargas R, Osorio F, Pereyra N: Eficacia de Leuprolide 11,25 mg para supresión de testosterona en pacientes con cáncer prostático. Revista Chilena de Urología. 2011; 76 (2): 83.

12. Denis L, Carneiro de Moura J, Bono A, et al. Goserelin Acetate and Flutamide Versus Bilateral Orchiectomy: A Phase III Eortc Trial (30853). Urology. 1993;42(2):119-29.

13. Ministerio de Salud. Medidas especiales sobre compra de medicamentos oncológicos. Lima: MINSA; 2011.

14. Bootman J, Townsend R, McGhan W. Principles of pharmacoeconomics. 3ed. Whitney Harvey books; 2005. 
15. Sacristán J, Ortún V, Rovira J, Prieto L, García Alonso F. Evaluación económica en medicina. Med Clin (Barc). 2004; 122(10):379-82.

16. Baly A, Toledo M, Rodríguez F. La economía de la Salud, la eficiencia y el costo de oportunidad. Rev Cubana Med Gen Integr. 2001; 17(4) 395-8.

17. Prieto L, Sacristán J, Antoñanzas F, Rubio-Terrés C, Pinto J, Rovira J. Análisis coste-efectividad en la evaluación económica de intervenciones sanitarias. Med Clin (Barc). 2004;122(13):505-10.

18. Walley T. Pharmacoeconomic and economic evaluation of drug therapies. En: Du Soich P, Erill S, Orme M, editores. The IUPHAR Compendium of Basic Principles for Pharmacological Research in Humans [Libro en línea]. Irvine, CA: International Union of Basic and Clinical Pharmacology; 2004; 67-75. [Citado 2011 Mar 16]. Disponible en: http://www.iuphar.org/clin_hu.html
19. U.S. National Library of Medicine. Leuprolide Injection, [Sede Web], [Acceso 09 de agosto de 2013]. Disponible en: https:// medlineplus.gov/druginfo/meds/a685040.htm

20. U.S. National Library of Medicine. Goserelin Implant, [Sede Web], [Acceso 09 de agosto de 2013]. Disponible en: https:// medlineplus.gov/druginfo/meds/a601002.html

21. Mendoza P. Análisis costo-efectividad. En: Evaluación económica en Salud. Universidad Nacional Mayor de San Marcos; 1995: 47.

Conflicto de intereses: Los autores declaran no tener conflictos de interés.

Fuente de financiamiento: Autofinanciado. 